\title{
Aportes de la investigación a las buenas prácticas en el aula universitaria
}

\author{
Research contributions to good practices in the university classroom \\ Contribuições da pesquisa às boas práticas na aula universitária
}

DOI: http://dx.doi.org/10.21803\%2Fpenamer.11.21.524

Blanca Aurelia Franzante Orcid: 0000-0001-6255-3573.

Marina Isabel Pagani Orcid: 0000-0001-5396-0740

José Manuel Perdomo Vázquez Orcid: 0000-0002-1655-7474

Liset Perdomo Blanco

\section{¿Cómo citar este artículo?}

Frazante, B., Pagani, M., Perdomo, J. M. \& Perdomo, L. (2018). Aportes de la investigación a las buenas prácticas en el aula universitaria. Pensamiento Americano, 11(21), 60-70

DOI: http://dx.doi.org/10.21803\%2Fpenamer.11.21.524

\begin{abstract}
Resumen
El presente artículo describe, en forma sucinta, resultados de investigaciones en el ámbito de la Educación Superior, desarrollados por docentes investigadores de la UCU- Argentina y UCLV- Cuba, cuya característica principal es el trabajo bajo la modalidad "en línea", en tanto, la distancia entre ambas universidades difiere por más de $6000 \mathrm{~km}$. Iniciado los intercambios hace un poco más de dos años, y una vez acordado los temas de interés se planteó desarrollar el primer trabajo de investigación sobre estilos docentes y grupos de investigación en línea. Durante su implementación, y tal como sucede en los caminos de la investigación, surgen nuevas preguntas, otros problemas, que permitieron plantear otros temas referidos por un lado a la Orientación Educativa y las estrategias de aprendizaje en el contexto universitario y las buenas prácticas en la docencia del nivel superior.

Sendos trabajos se plantean desde un enfoque cualitativo, por considerar que el mismo permite la comprensión del fenómeno que se desea estudiar desde una perspectiva holística, "desde adentro", involucrando a los diferentes actores del contexto estudiado.
\end{abstract}

PALABRAS CLAVES: Investigación- Educación Superior- Orientación Educativa- Buenas Prácticas

\begin{abstract}
This article describes, succinctly, the research results in the field of Higher Education, developed by researchers of the UCU-Argentina and UCLV-Cuba whose main characteristic is the blender learning used by both universities that are distanced more than $6000 \mathrm{~km}$.

The exchanges started a little over two years ago, and once the topics of interest were agreed upon, the first research work on teaching styles and online research groups was proposed. During its implementation, and as it happens in the ways of the investigation, new questions arouse, other problems, that allowed to raise other subjects referred on the one hand to the Educational Orientation and the strategies of learning in the university context and, on the other hand, the good practices in the superior level teaching.

The two works are proposed from a qualitative perspective, considering that it allows the understanding of the phenomenon that one wishes to study from a holistic perspective, "from within", involving the different actors of the studied context.
\end{abstract}

KEYWORDS: Research - Higher Education - Educational Guidance Good Practices. 


\section{Resumo}

O presente artigo descreve, de forma breve, os resultados de pesquisas no âmbito da Educação Superior, desenvolvido por docentes pesquisadores da UCU - Argentina e UCLV- Cuba, cuja característica principal é o trabalho sob a modalidade "em linha", uma vez que a distância entre as duas universidades é de mais de $6000 \mathrm{~km}$.

Os intercâmbios foram iniciados há pouco mais de dois anos, e uma vez estabelecido de comum acordo os temas de interesse, se propuseram a desenvolver o primeiro trabalho de pesquisa sobre estilos docentes e grupos de pesquisa em linha. Durante a sua implementação, e como se passa nos caminhos da pesquisa, surgem novas perguntas, outros problemas, que permitiram propor outros temas referidos por um lado à Orientação Educativa e às estratégias de aprendizagem no contexto universitário e as boas práticas na docência de nível superior. Ambos os trabalhos se desenvolvem desde um enfoque qualitativo, por considerarmos que o mesmo permite a compreensão do fenômeno que se deseja estudar desde uma perspectiva holística, "desde dentro", envolvendo os diferentes atores do contexto estudado.

PALAVRAS CHAVE: Pesquisa- Educação Superior- Orientação Educativa- Boas Práticas.

\section{Perfil}

Master en Salud Mental. Psicopedagoga. Profesora Titular. Investigadora Cat. III. Facultad de Ciencias de la Comunicación y la Educación. Universidad de Concepción del Uruguay.

blancafranzante@hotmail.com

\section{Perfil}

Especialista en TIC y Neurociencias. Investigadora novel. Universidad de Concepcion del Uruguay-Argentina. paganimarina@gmail.com

\section{Perfil}

Doctor en Ciencias Pedagógicas en el IPEK, Ucrania, (antigua URSS), Profesor de Física, Metodología de la Enseñanza de la Física, Pedagogía, Investigación Pedagógica. Miembro de Programas de Maestrías y de doctorado en el IPLAC, UCLV, ISPFV. Investigador. Universidad Central "Marta Abreu" de Las Villas. Cuba perdomo@uclv.edu.cu

\section{Perfil}

Dra. En Ciencias Pedagogicas. Investigadora. Universidad Central "Marta Abreu" de Las Villas. Cuba. lisetpb@uclv.cu

\section{Blanca Aurelia Franzante}

Psicopedagoga en Salud Mental.

\section{Marina Isabel Pagani}

Profesora en biología.

\section{José Manuel Perdomo V.}

Profesor superior de física y áreas de la educación.

\section{Liset Perdomo Blanco}

Licenciada en educación, profesora titular. 


\section{Introducción}

E n el presente artículo, se muestran avances de sendos trabajos de investigación enunciados en el resumen, y desarrollados por un equipo de docentes investigadores argentinos-cubanos.

Las diferentes técnicas de recolección de información utilizadas en los procesos investigativos permiten una triangulación de datos que sustentan los diagnósticos de problemáticas, como fuentes para las acciones de formación continua del profesor universitario.

A su vez cabe destacar la inherente formación del equipo de investigación en los procesos de trabajo "en línea", cuestión ésta poco desarrollada en investigaciones en el nivel superior. Si bien no es objeto de esta presentación este último punto, mencionar la importancia del trabajo en línea resulta oportuno pues ha permitido, a través de la sistematización de las actividades realizadas:

- Comprobar que es posible investigar en contextos diferentes y distantes, siempre que se reúnan determinados requisitos, de modo tal que los impedimentos tecnológicos y las distancias geográficas no limiten el desarrollo de nexos e intercambios entre los docentes investigadores.

- Desarrollar acciones de formación en los enfoques cualitativos en investigación educativa.

En el diseño del trabajo en línea para la investigación, y a partir de las inquietudes que emergen en el proceso de elaboración de resultados fue necesario manifestar las inquietudes, hacerse preguntas, saber sorprenderse $\mathrm{y}$ asombrarse ante hechos y situaciones que pasan desapercibidos, poder descotidianizar la realidad (Achilli, 2000). Estos saberes prácticos, emanados de la propia investigación, posibili- tan la conformación de habilidades ineludibles del investigador que desarrolla proyectos a distancia (Henriquez, 2013).

\section{Desarrollo}

Tal lo planteado, el enfoque metodológico cualitativo de las investigaciones tiene como particularidad el uso de distintas técnicas de recolección de datos, cuyo objetivo es abordar el objeto de estudio desde diferentes miradas, que puedan "reflejar la idiosincrasia y la complejidad del contexto que se estudia" (Vasilachis de Gialdino, 2007. p.30).

En este sentido, para una mejor comprensión de los procedimientos y resultados alcanzados en el trabajo que justifica la presente comunicación, es recomendable explicar la lógica seguida por los investigadores.

\section{¿Dónde se observa?: Las observaciones de clases y de diferentes espacios instituciona- les: biblioteca y patios donde transcurre la vida universitaria, ha sido un instrumento que se convierte en:}

- Un método de trabajo de indagación del quehacer diario de la universidad.

- Un proceso que debe ser planificado para recoger la información de la manera más exhaustiva posible.

- Un fin que facilita el conocimiento no solo descriptivo sino esencialmente comprensivo del contexto en general, y educativo en este caso.

A su vez, se reafirman tres principios, que a nuestro criterio orientan la observación:

- La recolección de la máxima información con el mínimo número de ítems observables.

- La complementación de la observación visual por otras fuentes generadoras de información.

- El planteamiento de la retroalimentación, con el docente que colabora en el rol de sujeto observable para la reconstrucción de lo obser- 
vado y del estudiante a través de grupos de discusión.

\section{¿Qué se observa?:}

-La clase en el contexto universitario, lugar "donde los sucesos se producen, la enseñanza se transforma en acto, el aprender se provoca y genera" (Souto, 1996 p.119).

- Y como se ha expresado, también otros espacios institucionales como biblioteca, pasillos, patios, confitería, son observados considerando, en palabras de Fernández (1994), que en los mismos se establecen tramas de relaciones que despliegan la tarea institucional y condiciones que pueden afectar la calidad del proceso de enseñanza-aprendizaje. En los espacios institucionales se "generan sensaciones de comodidad o incomodidad, seguridad o peligro, potencialidad o carencia, y con ella comprometen la posibilidad o dificultad de los sujetos para encontrarse "disponibles" frente a las demandas del trabajo (p. 22).

En forma sintética se describen ejemplos que permiten ilustrar lo reseñado:

Observación $\mathbf{N}^{\circ} 3$ : Realizada en la biblioteca durante la mañana

"Ocupación del lugar: 9 mesas ocupadas: 6 con 3 a 5 miembros; 2 con un alumno y, 1 con un profesor

Materiales en las mesas: Libros, apuntes, fotocopias, cuadernos, marcadores, resaltadores. Calculadoras científicas. No se usan computadoras personales (PC) de la biblioteca, algunos tienen notebook personal. Celulares en todas las mesas. La mayoría tiene mate (infusión típica de Argentina).

\section{Acciones:}

En grupo: Leen en voz baja, luego comentan los textos y "se explican". Hacen diagramas y explican.

En grupos y los que están solos: Subrayan y resaltan los textos. Hacen comentarios en los márgenes. Toman notas en cuadernos. Van a buscar agua para el mate.

profesor: busca libros en el mostrador. Revisa los textos y toma nota".

Grupo de la carrera de Educación Física (cuatro alumnos varones): Despliegan un plano de una cancha de Vóleibol. Hablan del juego. Comentan el Reglamento. Uno de ellos relata la experiencia del parcial, trabajan los errores que les ha señalado el profesor. Consideran las que están bien, pero también en algunas no están de acuerdo. Luego de la revisión del tema se retiran.

\section{Observación de una clase}

Por una cuestión de espacio, en el presente artículo se expresan sólo los indicadores tenidos en cuenta al observar una clase. "Indicadores, entendidos, como indicio o señal que permite el reconocimiento e identificación de evidencias (teóricas o empíricas) que han sido enmarcados en los momentos de una clase, quizás con enunciados tradicionales, pero que permiten un lenguaje que consideramos común en el ámbito educativo: Apertura, Desarrollo y Cierre". (Franzante et.al. 2015: s/n)

A modo de ejemplo se mencionan los ejes sobre los cuales se despliegan los indicadores en las actividades observadas:

-Momento de Apertura: ámbito físico. Clima inicial. Presentación. Diagnóstico inicial. Motivación inicial. Orientación hacia los objetivos de la clase.

-Momento de Desarrollo: motivación del segundo momento. Clima educativo. Nivel científico, técnico y actualidad de los contenidos. Información brindada a los estudiantes. Desarrollo de habilidades intelectuales, sociales, afectivas en la clase. Utilización de los me- 
dios de enseñanza aprendizaje. Tratamiento didáctico al error. Acciones espontáneas.

-Momento de Cierre: la evaluación. Cumplimiento de los objetivos propuestos. La anticipación para la motivación futura: "la tarea". Algunos indicadores "transversales". Características profesionales del docente.

El uso de múltiples técnicas de recolección de información permite complementar los datos emanados de las observaciones, con narrativas de su clase por parte del docente y sesiones de retroalimentación: docenteinvestigador. Con los estudiantes: grupos de discusión y charlas a través del chat.

Las narrativas, son pensadas como dispositivo documental centrado en la reflexión y análisis de relatos pedagógicos, los cuales den cuenta de las estrategias y prácticas profesionales de formación permanente. A partir de ello se considera que las narraciones re-construyen desde siempre nuestra historia de vida, el lenguaje como facultad humana permite re-presentar el mundo una y otra vez, ya que cada emisor hace presente, mediante la palabra y la escritura, algún aspecto de vida o su experiencia personal; en tanto el receptor actualiza ese saber y lo hace propio, en muchos casos, internalizando propuestas, modificando las percepciones y organizando un espacio en constante devenir histórico.Así en palabras de Suárez et. al (2005), podemos señalar que conversar con los docentes supone escuchar historias, "sumergirnos en relatos que narran experiencias escolares y las sutiles percepciones de quienes las viven, una oportunidad para comprender e introducirnos en el universo de prácticas individuales o colectivas que recrean vívidamente, con sus propias palabras, en un determinado momento y lugar, el sentido de la escolaridad" (p. 9).
Narración $\mathbf{N}^{\mathbf{a}}$ 8: A modo de ilustración se transcriben párrafos de una narración.

"La verdad es que me encanta la docencia y la investigación y creo que ambas van de la mano. Siempre trato de ponerme en el lugar del alumno... En mi paso por la universidad tuve buenas y malas experiencias... Docentes a los que siempre voy a recordar y otros a los que prefiero olvidar (...) Por eso conozco e intento conocer al alumno. Respeto a los que trabajan y trato de facilitarles las cosas (no les regalo nada)... Tengo colegas que pareciera que se olvidaron que fueron alumnos porque dan clases y pareciera que su intención es bocharlos. Repito, no les regalo nada, pero quiero que aprendan.

Siempre reconozco y aclaro que no sé todo, por cuestiones lógicas (...) Entonces de lo que sísé, trato de darles lo que más puedo, y busco e investigo aún más de lo que no sé..." (Prof. Mujer.)

A partir de lo expuesto $y$, respecto a la función de la narración en la investigación, se puede señalar junto a Porta y Yedaide (2014), que la universidad argentina ha recuperado parte del itinerario realizado por investigadores en educación resaltando la potencia del método biográfico, de las narrativas, como nuevas formas para comprender los procesos implicados en la docencia y la investigación en el nivel superior.

En este sentido el análisis de las observaciones y narraciones permiten reconocer las trayectorias docentes y la influencia que las mismas tienen en la práctica docente. Por ende, cómo cada profesor brinda "pistas", "señas" de lo que considera el estudiante debe aprender.

La universidad, puede ser un nicho de oportunidades que deben ser bien aprovechadas por los alumnos y profesores, los alumnos adquirirán lo que necesitan a partir de la experiencia en 
los caminos transitados por otros (sus docentes), mientras que los profesores deberán descubrir las necesidades no solamente de conocimientos y habilidades sino también las afectivas y espirituales de sus estudiantes para acompañar el proceso de enseñanza- aprendizaje (Monsalve Lozano, 2010).

Sesiones de retroalimentación, siguiendo a Sirvent (2006), se considera que las mismas son "espacios de convergencia de diferentes fuentes de información que posibilitan la construcción y validación del conocimiento. Pueden ser consideradas situaciones de "triangulación metodológica in situ", en la medida que estamos combinando diferentes metodologías en el estudio del mismo fenómeno en un espacio de confrontación" (p.s/n). Agrega la autora, que la sesión de retroalimentación no es una devolución sino un espacio de construcción colectiva de conocimiento donde se conjuga la investigación, participación y aprendizajes mutuos.

En este sentido, como cierre del trabajo con el docente en una sesión posterior trabajamos en forma conjunta el material que aporta el docente a través de su narrativa y el investigador los indicadores observados.

\section{Sesiones de retroalimentación. Párrafos ilus- trativos}

"Noto, como siempre, que es una costumbre por parte de los estudiantes sacar apuntes y hacer pocas preguntas, es decir, que si pueden pasar desapercibidos mejor. El ida y vuelta tuvo que ser iniciado por mi parte" (Sesión Nº 4. Profesor)

“...no estamos preparados para colaborar con las estrategias de aprendizaje de los alumnos actuales" (Sesión N6, Profesor)

Estas sesiones se convierten en un proceso de intercambio de información que aporta tanto a la práctica docente como al proceso de investigación, y a las acciones de formación.
Con los estudiantes tal lo señalado, además de las observaciones, se aborda sus percepciones del proceso de enseñanza -aprendizaje a través de grupos de discusión y conversaciones a través del chat.

Los Grupos de discusión, son encuadrados en la familia de entrevistas grupales orientadas a la obtención de información cualitativa. El mismo "no es equiparable a ninguna de sus modalidades próximas: no es una conversación grupal natural, no es un grupo de aprendizaje como terapia psicológica (...), tampoco es un foro público...; sin embargo, parasita y simula (parcialmente), a la vez, cada una de ellas" (Canales y Peinado, 1994:292 citado en Valles 2003). Enfatiza el autor que dicha "definición comparada sintetiza las reflexiones metodológicas de diversos autores (Ávila Espada y García de la Hoz, 1994; e Ibáñez, 1981,1991; entre otros) y subraya lo específico y lo compartido de esta técnica con respecto a otras modalidades grupales, ordinarias o profesionales". (Citado en Valles, M. 2003. p 294)

Se puede agregar que otra de las características tenidas en cuenta para la selección de esta técnica se basó en el estar ligada al concepto de interacción, en tanto la situación grupal abre la oportunidad de escucharse y dialogar sobre las propias experiencias (en este caso de aprendizaje), en un contexto donde dichas experiencias adquieren significación. La situación de grupo hace que las respuestas o intervenciones surjan como la reacción a las respuestas o intervenciones de otros miembros presentes en la reunión, produciéndose un efecto de sinergia.

\section{Grupos de discusión. Ejemplos ilustrativos}

“...No, no fui aprendiendo sola... (Mujer. E.3); "...al principio en el ingreso tuvimos un curso y donde nos daban algunas estrategias y técnicas de estudio... nos dijeron que nos iban a ayudar en toda la carrera, pero nunca más se acercaron... nos enseñaron a hacer cuadros, resúmenes, pero después que- 
da todo en el nivel de cada uno... (Varón. E.7)"

Charlas vía chat, fue propuesta por los propios estudiantes. Frente al pedido de continuidad de la entrevista si surgían dudas al desgrabar y escuchar la misma, ellos proponen esta modalidad. Realmente ha sido una experiencia muy enriquecedora en tanto las respuestas son "casi" inmediatas, y sin dificultades. Si tenemos en cuenta que los jóvenes de hoy son "nativos digitales", estas nuevas formas de comunicación deberían ser tenidas en cuenta al momento de elegir técnicas complementarias de recolección de datos.

Así, se puede resumir lo reseñado que las diferentes formas de comunicación tanto con docentes como con estudiantes permitieron, junto con el registro de los estilos de enseñanza de los docentes y de aprendizaje de los estudiantes, identificar en forma explícita e implícita la necesidad de abordar aquellas cuestiones que se relacionan con las estrategias de enseñanza-aprendizaje en el nivel superior y la función de orientador del profesor en la elaboración de las mismas.

El papel que desempeñaron y desempeñan las tecnologías de la información y las comunicaciones (TIC) en los alumnos y docentes, favoreció el abordaje de un amplio abanico de estudios sobre la complejidad que brinda el uso de las mismas y la experiencia profesional recorrida. Las revoluciones tecnológicas han representado momentos fundamentales para la transformación del mundo en que vivimos. En cada una de ellas ha sido posible rebasar los límites cognitivos impuestos por cada época sobre la manera de pensar y de realizar acciones que pudiesen transformar la relación del ser humano con su entorno (Martinelli Ramírez y Casillas, 2015).

Desde las tecnologías de la información y la comunicación, se puede argumentar que las "re- des" han tenido un gran impacto social en diferentes espacios de la investigación educativa, favoreciendo el pasaje de ser lectores a ser autores, a trabajar y aprender en forma colaborativa, a integrar redes sociales, entre muchas otras posibilidades. Los nuevos recursos facilitaron instancias de producción de contenido desde una variedad de lenguajes. Si bien Internet no fue creada con un fin pedagógico, es el andamiaje que permitió a docentes investigadores y alumnos, la posibilidad de construcción de nuevos conocimientos, a través de su utilización (Velázquez, 2012).

El análisis de los datos recogidos favorece, mientras se desarrolla la investigación proponer diferentes acciones que convierten a los investigadores en orientadores. Entendiendo a la orientación como

"una intervención para lograr unos objetivos determinados enfocados preferentemente hacia la prevención, el desarrollo humano y la intervención social. Dentro del desarrollo se incluye el auto-desarroIlo, es decir, la capacidad de desarrollarse a sí mismo como consecuencia de la auto-orientación. Esto significa que la Orientación se dirige hacia el desarrollo de la autonomía personal como una forma de educar para la vida". (Bisquerra, 2005. p.1)

Y a su vez, como un proceso continuo que involucra a todos los actores del proceso educativo: docentes, equipo de gestión, técnico, investigador, en suma "todo aquél que participe en la comunidad educativa y pueda oír, atender y comprender lo que otro/s dice/n, desde un respetuoso silencio para comprender los mensajes, el pedido, para que circule la pregunta y facilite el diálogo y la producción de pensamiento" (Ianni, 2003.p.59) y el que se presenta a continuación o sea el de la orientación educativa en el nivel superior. 


\section{La Orientación Educativa en el nivel superior}

Señala Vuelvas Salazar (2004) que es posible "investigar en Orientación Educativa porque no se trata de un contenido de orientación para la intervención, tampoco una teoría que sustente un tipo de práctica, sino de un enfoque que tiene por objeto la construcción social de la realidad y han sido Berger y Luckmann (1998) quienes lo han elevado al rango de categoría de investigación" (citado en Vuelvas Salazar 2004: s/n)

En este sentido, y teniendo en cuenta que el equipo de investigación está conformado por docentes investigadores de las propias instituciones involucradas, surge la necesaria reflexión sobre el momento en que deberían abordarse los resultados. En tanto se considera que los procesos investigativos se desarrollan paralelos a la actividad cotidiana universitaria, los resultados parciales pueden ser indicadores que permiten implementar acciones de transferencia a través de espacios de co-formación. ¿Por qué de formación conjunta? Porque los ejes que se proponen de formación surgen del análisis e interpretación de la observación de las clases, narraciones, sesiones de retroalimentación, grupos de discusión, lo cual se convierte en una devolución del trabajo de los investigadores a sus propios colegas. Ello permite analizar si la lectura realizada coincide con las necesidades no sólo del docente, sino también del destinatario último de las acciones propuestas: "el estudiante". Es importante considerar que en los talleres de orientación a docentes se potencia el empleo de métodos y técnicas propios de la orientación educativa (Monsalve Lozano, 2010).

\section{El docente orientador}

Siguiendo a Ayala (citado en Molina Contreras 2004), se puede señalar que la orientación académica implica "un proceso de asesoramiento continuo donde el docente promueve actividades de tipo preventivo dirigidas a la formación de hábitos de estudio, atención y concentración en clase, aprovechamiento del tiempo y desarrollo de habilidades cognitivas". Desde el punto de vista de la relación enseñanza-aprendizaje, la orientación escolar refiere a un "proceso dirigido al desarrollo de habilidades y destrezas para aprender a aprender y formar hábitos, actitudes, valores y comportamientos positivos hacia el medio escolar en relación a las actividades e aprendizaje." (Molina Contreras. 2004. p.6)

En este sentido, en el próximo apartado se describen en forma sucinta diferentes acciones de formación emanadas del desarrollo del proyecto.

\section{Síntesis de las actividades implementa- das y por implementar y sus aportes.}

Teniendo en cuenta lo expresado a lo largo del trabajo y para finalizar el desarrollo del mismo, se resumen diferentes acciones de formación y acompañamiento al docente, emanadas de los resultados que se van obteniendo en los proyectos. Se considera que la investigación en el aula puede ser una fuente para el desarrollo de buenas prácticas en la docencia, en tanto permite orientar la actividad del profesor y los alumnos a partir de visiones externas fundamentadas no sólo desde la práctica, sino también desde la teoría.

\section{a-Seminario Taller "Estrategias de ense- ñanza- aprendizaje en el Nivel Superior" (UCU-Implementado)}

El mismo tuvo como objetivo generar espacios de reflexión acerca del papel de orientador del docente en la elaboración de estrategias de enseñanza- aprendizaje.

La propuesta de trabajo desde una metodología de seminario- taller implica un espacio de co-aprendizaje, donde los aportes de los especialistas se ven enriquecidos con la interacción y análisis de la experiencia áulica de los participantes.

Las clases se implementan abordando la te- 
mática desde "demostraciones" por parte del docente aplicando diferentes estrategias y luego, un espacio grupal donde se re- trabajan los conceptos de forma participativa con trabajos cooperativos.

A lo largo de año académico, los docentes comunican aquellas estrategias que van implementando, los aspectos positivos y los obstáculos. A partir de ello se planifican nuevos encuentros de intercambio y evaluación de las propuestas.

\section{b- Aportes de la investigación a las buenas prácticas en el aula universitaria (UCLV-Imple- mentado)}

En el caso de la UCLV se registran varios resultados en el desarrollo de las actividades del Proyecto institucional de Innovación tecnológica "Experiencias, estilos y buenas prácticas en la docencia universitaria" (Proyecto 10049) que tiene por objetivo general: Valorar las experiencias de los docentes experimentados para sustentar los modelos de actuación profesional en el proceso docente educativo en la Universidad, desarrollado por profesores del Centros de Estudio de Educación.

Además de las contribuciones individuales de los docentes que pertenecen al referido proyecto se han realizado talleres para el intercambio de experiencias con docentes de trayectoria. Las narraciones de los docentes participantes contribuyen a establecer modelos de actuación profesional para aplicar en la docencia.

En las conclusiones de los talleres se presentan recomendaciones importantes para la superación profesional con el fin del mejoramiento de la docencia universitaria.

\section{c- Taller "Experiencias en el empleo de la in- vestigación cualitativa en educación" (UCU- UCLV- Implementado)}

El taller tuvo como objetivo trabajar con docen- tes conceptos de la investigación cualitativa en educación, a partir del trabajo de campo desarrollado en los proyectos de investigación que se resumen en la presente ponencia.

Temas abordados: Investigación educativa. Formación docente. Características particulares de la investigación cualitativa. Diseño de la investigación cualitativa. Estrategias de investigación cualitativa. Técnicas cualitativas. Análisis de datos: el método comparativo constante.

\section{d- Curso de Formación permanente y conti- nua. "Una alternativa para la formación do- cente continua de docentes en la Educación Superior"}

Esta actividad tuvo como objetivo principal, incorporar a la formación inicial recibida sustentos teóricos, metodológicos y visiones para el reconocimiento de la necesidad de una formación continua en el ejercicio del quehacer docente de la educación superior.

Las temáticas abordadas refirieron a la problemática de la educación superior en los contextos actuales y el curriculum; la incorporación de las tecnologías de la información y comunicación al aula universitaria y la investigación educativa como un espacio inherente a la función del docente universitario. A medida que las mismas se desarrollaron surgieron nuevas solicitudes de los docentes que participan y de aquellos que reciben información sobre las mismas, las cuáles se canalizaron en otra actividad: "conversatorios sobre Buenas Prácticas"

Lo desarrollado en los diferentes apartados son recortes que pretenden "mostrar" el camino recorrido hasta el momento donde se integra la docencia, la investigación y la extensión, funciones inherentes al profesor universitario.

\section{Cierre... a modo de reflexiones para seguir pensando}

Los aportes de la investigación a las buenas 
prácticas docenes en el aula universitaria tienen un camino recorrido relativamente amplio y diverso, pero sus contribuciones se manifiestan además en forma recíproca dado por el hecho de que las mismas también pueden propiciar aportes tanto a la investigación como a los propios docentes investigadores.

El trabajo de campo del proyecto de investigación, el análisis, interpretación y conceptualización de los datos recolectados, colaboran al mejoramiento de la docencia universitaria al menos de aquellos profesores que participan de las diferentes actividades, convirtiéndose las mismas en una fuente genuina de formación. Una formación que tiene como eje la co-formación, en tanto las actividades grupales y/o individuales propuestas estimulan la reflexión y análisis de la práctica áulica, generándose espacio donde el profesor revisa, cuestiona y se interroga sobre las mismas. Ello se transforma en fuente de conocimiento no sólo del quehacer docente sino también de las implicancias en las mismas y en la conformación de su identidad como profesor universitario.

La utilización de la red como estrategiapara el trabajo "en línea", permitió y permite al equipo de investigación "re" conocerse en nuevas formas de afrontar la formación continua en el campo de la investigación educativa en el nivel superior de educación.

A su vez, se puede agregar que el intercambio entre los docentes investigadores ha permitido hacer una exploración mucho más profunda sobre realidades, potencialidades y necesidades no sólo de las experiencias en investigación sino también en la docencia universitaria. Como equipo, se ha podido constatar que la fortaleza de un grupo de investigación, al menos en el campo educativo, está no solamente en el intercambio bibliográfico, en los ejemplos en la solución de problemas y las formas de enfrentar dificultades, sino también en las trans- formaciones que se producen en los propios investigadores durante la adquisición de nuevas miradas en el enfrentamiento a estas dificultades lo cual genera a la vez un torrente de iniciativas y la posibilidad de realizar acciones conjuntas como complemento a los esfuerzos que se realizan en la práctica cotidiana, así como el despliegue de alternativas no consideradas anteriormente por encontrarnos en un "círculo cerrado en la investigación"

Otros de los aportes que se considera importante, generado en el trabajo investigativo "a distancia" está en el propio re-conocimiento que realizan los investigadores entre sí para complementar el trabajo. Un ejemplo de ello está en la propia conformación de la presente ponencia la cual no ha seguido los cánones tradicionales y los estilos estereotipados de elaboración revisión y presentación.

Al equipo de investigación nos ha quedado la satisfacción como producto implícito de la labor realizada de investigar a distancia mediante la generación de intercambios "en línea", señalar que una realidad distinta a la acostumbrada en la solución de problemas educativos es posible siempre que se generen espacios donde primen la sinceridad científica, la ética profesional y el reconocimiento de las posibilidades del otro.

Este trabajo ha permitido además generar un grupo de nuevas ideas que seguramente presentaremos en oportunidades posteriores como, por ejemplo: a) Modelo para la formación de investigadores en diferentes contextos, b) Una estrategia de trabajo investigativo en el campo educativo, c) Experiencias en proyecto investigativo "a distancia" a partir de los nuevos recursos de las tecnologías de la información y las comunicaciones. 


\section{Referencias}

Achilli, E. (2000). "Investigación y Formación docente". Laborde Editor.Rosario.

Bisquerra, R. 2005. "Marco Conceptual de la Orientación Psicopedagógica". Revista Mexicana de Orientación Educativa, Vol. III Nº, México- 2-8

Fernández, L. (1994). "Instituciones educativas. Dinámicas institucionales en situaciones Críticas". Paidós. Bs. As. 1994.

Franzante, B., Perdomo Blanco, L., Rodríguez Díaz, R. (2015). Ponencia: "Indicadores para la identificación de estilos y modelos que subyacen en las prácticas docentes en una universidad argentina y una universidad cubana". III Congreso Internacional "Universidad, sociedad y futuro. Hacia una nueva reforma universitaria en América Latina". Buenos Aires.

Henríquez Fuentes, G. R. (2013). Investigación cualitativa en modelos de gestión logística y sus estrategias de desarrollo en la costa Caribe colombiana. Ad-Gnosis, 2(2), 65-85

Ianni, N. D. (2003). "La orientación escolar en tiempos de incertidumbres: una tarea compleja y difícil, necesaria y posible". Ensayos y Experiencias $N^{\circ}$ 47. Ed. Novedades Educativas. Argentina. 53-60.

Martinelli Ramírez, A. y Casillas, M. Á. (2015). "Internet en Educación Superior". Colección Háblame de TIC 2. Editorial Brujas. Córdoba. Argentina.

Molina Contreras, D. L. (2004). "Concepto de orientación educativa: diversidad y aproximación". Revista Iberoamericana de Educación. Ed. OEl. N³3/6.

Monsalve Lozano, B. (2010). El emprendimiento y la universidad. Pensamiento Americano. 3(5) 51-54.

Porta, L. y Yedaide, M. M. (2014). "La investigación biográfico narrativa. Desafíos ontológicos para la investigación y la enseñanza en la formación de formadores". Sophia: Colección de Filosofía de la Educación. Buenos Aires.

Sirvent, M. T. (2006). "Los diferentes modos de operar en investigación social" y "El proceso de investigación, las dimensiones de la metodología y la construcción del dato científico". Cuadernos de publicaciones Facultad de Filosofía y Letras, Universidad de Buenos Aires. Buenos Aires.

Souto, M. (1996) "La clase escolar. Una mirada desde la didáctica de lo grupal" en Camilloni, A. et. al. 1996. Ed. Paidós. Buenos Aires.

Suárez, D.; Ochoa, L.y Dávila. P. (2005). “La documentación narrativa de experiencias pedagógicas. Hacia la reconstrucción de la memoria y el saber profesional de los docentes". Revista Nodos y nudos No78. Universidad Pedagógica Nacional Colombia.

Valles, M.S. (2003). "Técnicas cualitativas de investigación social. Reflexión metodológicay práctica profesional". Ed. Síntesis S.A. Madrid.

Vasilachis de Gialdino, I. (comp) (2007). "Estrategias de investigación cualitativa". Ed. Gedisa. Buenos Aires.

Velázquez, C. (2012). Estrategias Pedagógicas con TIC. Recursos Didácticos para entornos 1 a 1. Ediciones Novedades Educativas. Buenos Aires.

Vuelvas Salas, B. (2004). La investigación en Orientación Educativa. Revista Mexicana de Orientación Educativa. Vol. // Nº 3. México

Zabalza Beraza, M. (2012) "Buenas prácticas docentes en la enseñanza universitaria" Revista de Docencia Universitaria $\mathrm{Vol} 70 \quad N^{\circ}$. Disponible en: http:// redaberta.usc.es/redu/
2018, Vol. 11(21) 60-70 @ The Author(s) 2018 Reprints and permission: www.americana.edu.co https://www.coruniamericana.edu.co/publicaciones/ojs/index.php/pensamientoamericano/index 